\title{
Global well-posedness and exponential decay rates for a KdV-Burgers equation with indefinite damping
}

\author{
M.M. Cavalcanti ${ }^{\mathrm{a}, 1}$, V.N. Domingos Cavalcanti ${ }^{\mathrm{a}, 2}$, V. Komornik ${ }^{\mathrm{b}, *}$, J.H. Rodrigues ${ }^{\mathrm{a}}$ \\ a Departamento de Matemática, Universidade Estadual de Maringá, 87020-900, Maringá, PR, Brazil \\ b Département de Mathématique, Université de Strasbourg, 7 rue René Descartes, 67084 Strasbourg Cedex, France
}

Received 5 December 2012; received in revised form 17 July 2013; accepted 12 August 2013

Available online 13 September 2013

\begin{abstract}
We consider the KdV-Burgers equation $u_{t}+u_{x x x}-u_{x x}+\lambda u+u u_{x}=0$ and its linearized version $u_{t}+u_{x x x}-u_{x x}+\lambda u=0$ on the whole real line. We investigate their well-posedness their exponential stability when $\lambda$ is an indefinite damping. () 2013 Elsevier Masson SAS. All rights reserved.
\end{abstract}

MSC: primary 35Q53; secondary 93D15

Keywords: KdV-Burgers equation; Well-posedness; Stabilization by feedback; Decay rate

\section{Introduction}

The goal of this work is to prove the exponential stability of the Cauchy problem

$$
\begin{cases}u_{t}+u_{x x x}-u_{x x}+\lambda u+\alpha u u_{x}=0 & \text { in } \mathbb{R} \times(0, \infty), \\ u(0)=u_{0} & \text { in } \mathbb{R},\end{cases}
$$

where $\lambda \in L^{\infty}(\mathbb{R})$ is a function which is allowed to change sign and $\alpha$ is a constant which assumes $\alpha=0$ or $\alpha=1$. These assumptions made on $\alpha$ imply that we are considering both the linear and the nonlinear problem.

In this work we were inspired by the equation

$$
u_{t}+u_{x x x}-u_{x x}+u u_{x}=0 \quad \text { in } \mathbb{R} \times(0, \infty) .
$$

This equation gained some popularity when the necessity to attach dissipation to nonlinearity and dispersion arises in modelling unidirectional propagation of planar waves.

\footnotetext{
* Corresponding author. Tels.: +33 368850207 (office), +33 953695712 (home).

E-mail addresses: mmcavalcanti@uem.br (M.M. Cavalcanti), vndcavalcanti@uem.br (V.N. Domingos Cavalcanti), vilmos.komornik@math.unistra.fr (V. Komornik),jh.rodrigues@ymail.br (J.H. Rodrigues).

1 Research of Marcelo M. Cavalcanti partially supported by the CNPq Grant 300631/2003-0.

2 Research of Valéria N. Domingos Cavalcanti partially supported by the CNPq Grant 304895/2003-2.
} 
Here $u=u(x, t)$ is a real-valued function of two real variables $x$ and $t$, which in applications corresponds to the distance in the direction of propagation and to passed time, respectively. The dependent variable may represent a displacement of the underlying medium or a velocity, for example. Eq. (1.2) is referred to as Korteweg-de Vries-Burgers equation (KdVB equation) because it represents the union of the Korteweg-de Vries equation

$$
u_{t}+u_{x x x}+u u_{x}=0
$$

and the Burgers equation

$$
u_{t}-u_{x x}+u u_{x}=0 .
$$

Solutions of (1.2) should approach zero as $t$ goes to infinity. A natural question which arises is about the rate at which $\|u(t)\|$ approaches zero, where $\|\cdot\|$ is some norm for real-valued functions of a real variable. In [1] the authors prove that the solution of (1.2) corresponding to the initial data $u_{0} \in L^{1}(\mathbb{R}) \cap H^{2}(\mathbb{R})$ satisfies the inequality

$$
\|u(\cdot, t)\|_{L^{2}(\mathbb{R})} \leqslant C t^{-\frac{1}{4}}
$$

for all $t>0$ with some positive constant $C$. Moreover, this estimate is optimal because $\int_{\mathbb{R}} u_{0}(x) d x \neq 0$.

The study of decay of the energy associated with dispersive nonlinear equations is very interesting and a considerable number of researchers have determined significant advances to the development of this subject. In [4] the authors gave a new contribution with respect to the decay of the energy related to mild solutions for the damped Korteweg-de Vries $(\mathrm{KdV})$ type equation given by

$$
u_{t}+b u_{x}+u_{x x x}+u u_{x}+a(x) u=0,
$$

where $u=u(x, t)$ is a real-valued function, $b$ is a real constant and $a=a(x)$ is a non-negative function. In this paper, they consider the initial value problem (IVP)

$$
\begin{cases}u_{t}+b u_{x}+u_{x x x}+u u_{x}+a(x) u=0 & \text { in } \mathbb{R} \times(0, \infty) \\ u(x, 0)=u_{0}(x) & \text { for } x \in \mathbb{R}\end{cases}
$$

and the initial boundary value problem (IBVP)

$$
\begin{cases}u_{t}+b u_{x}+u_{x x x}+u u_{x}+a(x) u=0 & \text { in } \mathbb{R} \times(0, \infty), \\ u(0, t)=0 & \text { for } t \geqslant 0, \\ u(x, 0)=u_{0}(x) & \text { for } x \geqslant 0,\end{cases}
$$

where $\mathbb{R}_{+}=(0, \infty)$.

In both cases, the non-negative function $a(x)$ is responsible for the dissipative effect.

Eq. (1.5) is a generalization of the well-known $\mathrm{KdV}$ equation,

$$
u_{t}+b u_{x}+u_{x x x}+u u_{x}=0 .
$$

In the case of the initial value problem related to (1.8), the value $\|u(\cdot, t)\|_{L^{2}(\mathbb{R})}^{2}$ can be interpreted as the energy. It is obvious that for a smooth and decaying at infinity solution $u(x, t)$ to $(1.8)$, the energy is a constant of motion, that is

$$
\int_{\mathbb{R}} u^{2}(x, t) d x=\int_{\mathbb{R}} u_{0}^{2}(x) d x,
$$

so there is no decay of the energy as $t \rightarrow \infty$. On the other hand, if we consider $a(x) \geqslant \alpha_{0}>0, \forall x \in \mathbb{R}$, then the solution to Eq. (1.6) satisfies

$$
\int_{\mathbb{R}} u^{2}(x, t) d x \leqslant e^{-2 \alpha_{0} t} \int_{\mathbb{R}} u_{0}^{2}(x) d x .
$$

The main goal of this article was to establish the decay of the energy in the cases when $a(x) \not \equiv 0$ but it is not assumed the existence of a positive constant $\alpha_{0}$ such that $a(x) \geqslant \alpha_{0}$. It was the main novelty of this paper since in the previous results in the related literature, the function $a(x)$ was considered strictly positive at $\infty$. 
A first result on exponential decay of the energy for the IBVP (1.7) considering a localized damping was established in [16]. In this paper $a(x) \geqslant \alpha_{0}>0$ in $(0, \delta) \cup(R, \infty)$ for some $0<\delta<R$. In [19] it was pointed out that the interval $(0, \delta)$ can be dropped and it was proved the exponential decay of the energy in weighted spaces with exponential and power weights. The decay of spatial derivatives of solutions was also derived.

We remark that in [2] for the non-homogeneous IBVP (1.7), considering a constant damping $a(x)=\alpha_{0}>0$ and small but not decaying boundary data, the exponential decay at the $H^{k}$-level for $k=1,3$ or 4 is obtained (without restrictions on the size of initial data).

Taking into account the KdV equation posed on a finite interval with localized damping, the exponential decay of the energy was established in [17] and [18]. Considering KdV type equations with more general nonlinearities, these results were extended in [15] and [22] while periodic problems were studied in [10,11,14,24]. A good review regarding these topics is given in [23].

Note that for small initial and boundary data, exponential decay of the energy is obtained for KdV equation posed on a finite interval, without any damping. In addition, the internal dissipation is responsible for the validity of such result even for small anti-damping (see $[6,7,12,13,3]$ ). In [8] nontrivial stationary smooth solution to the KdV equation posed on a finite interval with zero boundary data is constructed.

In [9] the solutions to problem (1.8) with initial boundary conditions as in (1.7) and small initial data are considered and, the pointwise decay as $t \rightarrow \infty$ is established.

In spite of having many works dealing with the $\mathrm{KdV}$ equation in the existing literature, the same cannot be asserted to the $\mathrm{KdV}$-Burgers equation. This lack of results becomes more evident when we are interested in the asymptotic behaviour of its solutions. In this context, we can cite the article [5], where the author used global attractors theory in order to study the asymptotic behaviour at the $H^{2}(\mathbb{R})$-level of the semigroup associated to the generalized $\mathrm{KdV}$-Burgers equation

$$
u_{t}+\left(\delta u_{x x}+g(u)\right)_{x}-v u_{x x}+\gamma u=f(x), \quad t>0, x \in \mathbb{R}
$$

where $\delta, v>0$ and $\gamma \geqslant 0$ are constants, $f \in H^{2}(\mathbb{R})$, and $g$ is a Lipschitz function of class $C^{2}(\mathbb{R})$.

The solutions to the considered problems, regarded in the distributional sense, are called weak solutions. Weak solutions on a time interval $(0, T)$ are called mild solutions if $u(\cdot, t) \in C\left([0, T] ; H^{1}(I)\right)$ and $u u_{x}+a u \in L^{2}\left(0, T ; L^{2}(I)\right)$, where either $I=\mathbb{R}$ or $I=\mathbb{R}_{+}$. The main reason to select such a class of solutions from the set of weak solutions is that they can be regarded as solutions to the corresponding linear problems

$$
u_{t}+b u_{x}+u_{x x x}=f(x, t)
$$

where $f \in L^{2}\left(0, T ; L^{2}(I)\right)$.

This article is organized as follows. In Sections 2 and 3 we investigate the well-posedness and stability of the corresponding linear problem. Sections 4, 5 and 6 are dedicated to the nonlinear problem. Under the effect of an indefinite damping mechanism, global well-posedness and exponential stability results are established.

\section{Well-posedness for the linear problem}

In this section we consider the following problem:

$$
\begin{cases}u_{t}+u_{x x x}-u_{x x}=0 & \text { in } \mathbb{R} \times(0, \infty) \\ u(x, 0)=u_{0}(x) & \text { for } x \in \mathbb{R}\end{cases}
$$

The next result ensures that this problem is well posed in $L^{2}(\mathbb{R})$.

Proposition 2.1. The operator $A:=-\partial_{x}^{3}+\partial_{x}^{2}$ defined on $D(A):=H^{3}(\mathbb{R})$ generates a semigroup in the Hilbert space $H:=L^{2}(\mathbb{R})$. 
Proof. According to the Lumer-Phillips theorem it is sufficient to check that $A$ is dissipative and that $I-A$ is onto.

The dissipativity follows by a direct computation: if $u_{0} \in H^{3}(\mathbb{R})$ is real-valued, then $u$ is also real-valued and

$$
\begin{aligned}
(A u, u)_{H} & =\int_{-\infty}^{\infty}\left(-u_{x x x}+u_{x x}\right) u d x \\
& =\int_{-\infty}^{\infty} u_{x x} u_{x}-u_{x}^{2} d x \\
& =\frac{1}{2} \int_{-\infty}^{\infty} \frac{d}{d x} u_{x}^{2} d x-\int_{-\infty}^{\infty} u_{x}^{2} d x \\
& =-\int_{-\infty}^{\infty} u_{x}^{2} d x \\
& \leqslant 0 .
\end{aligned}
$$

Since $\Re A v=A(\Re v)$ for all $v \in H^{3}(\mathbb{R})$, it follows that

$$
\Re(A u, u)_{H}=-\int_{-\infty}^{\infty}\left|u_{x}\right|^{2} d x \leqslant 0
$$

for all $u_{0} \in H^{3}(\mathbb{R})$.

It remains to show that for every $f \in L^{2}(\mathbb{R})$ there exists $u \in H^{3}(\mathbb{R})$ satisfying the equality

$$
u-u_{x x x}+u_{x x}=f .
$$

Taking the Fourier transform it is equivalent to

$$
\hat{u}(\xi)\left(1-(i \xi)^{3}+(i \xi)^{2}\right)=\hat{f}(\xi)
$$

or to

$$
\hat{u}(\xi)=\frac{\hat{f}(\xi)}{1-(i \xi)^{3}+(i \xi)^{2}} .
$$

In the last step we have used the fact that the denominator

$$
h(\xi):=1-(i \xi)^{3}+(i \xi)^{2}
$$

never vanishes. Since, moreover, $h(\xi)$ is a continuous function satisfying $|h(\xi)| \rightarrow \infty$ as $|\xi| \rightarrow \infty, 1 / h$ is bounded, and therefore the last equation has a unique solution $\hat{u} \in L^{2}(\mathbb{R})$.

Finally, since the function

$$
\frac{1+|\xi|+|\xi|^{2}+|\xi|^{3}}{\left|1-(i \xi)^{3}+(i \xi)^{2}\right|}
$$

tends to 1 as $|\xi| \rightarrow \infty$ and hence it is bounded by some constant $M$ on $\mathbb{R}$, we conclude that

$$
\left|(i \xi)^{j} \hat{u}(\xi)\right| \leqslant M|\hat{f}(\xi)|, \quad j=0,1,2,3 .
$$

Since $\hat{f} \in L^{2}(\mathbb{R})$, this implies the regularity property $u \in H^{3}(\mathbb{R})$. 


\section{Exponential decay rates for the linear problem}

The proof of the dissipativity in the preceding section shows that the strong solutions of (2.1) (for which $u_{0} \in H^{3}(\mathbb{R})$ ) satisfy the relation

$$
\frac{d}{d t} \int_{-\infty}^{\infty}|u(x, t)|^{2} d x=-2 \int_{-\infty}^{\infty}\left|u_{x}(x, t)\right|^{2} d x \leqslant 0 .
$$

This is, however, not sufficient for the exponential stability because the nonzero constant functions solve (2.1).

In order to ensure the exponential stability of the KdV-Burgers equation in $H:=L^{2}(\mathbb{R})$, we thus need some additional damping mechanism. Inspecting the proof of Proposition 2.1, it is natural to consider the following modified problem:

$$
\begin{cases}u_{t}+u_{x x x}-u_{x x}+\lambda u=0 & \text { in } \mathbb{R} \times(0, \infty), \\ u(x, 0)=u_{0}(x) & \text { for } x \in \mathbb{R},\end{cases}
$$

where $\lambda$ is some given non-negative function.

Proposition 3.1. If $\lambda \in L^{\infty}(\mathbb{R})$, then the operator $A_{\lambda}$ defined by the formula $A_{\lambda} u:=-u_{x x x}+u_{x x}-\lambda u$ on $D\left(A_{\lambda}\right):=$ $H^{3}(\mathbb{R})$ generates a semigroup in the Hilbert space $H:=L^{2}(\mathbb{R})$.

Proof. It suffices to observe that $A_{\lambda}$ is a bounded perturbation of the operator $A$ of Proposition 2.1 and therefore it is also the infinitesimal generator of a semigroup.

Next we prove the following

Proposition 3.2. If $\lambda \in L^{\infty}(\mathbb{R})$ has a positive lower bound $\lambda^{\prime}$, then the problem (3.2) is exponentially stable and its solutions satisfy the decay estimates

$$
\|u(t)\|_{L^{2}(\mathbb{R})} \leqslant e^{-\lambda^{\prime} t}\left\|u_{0}\right\|_{L^{2}(\mathbb{R})} \quad \text { for all } t \geqslant 0
$$

Proof. If $u_{0} \in H^{3}(\mathbb{R})$, then repeating the proof of (2.2) and (3.1) with $A$ replaced by $A_{\lambda}$ we obtain that

$$
\begin{aligned}
\frac{d}{d t} \int_{-\infty}^{\infty}|u|^{2} d x & =-2 \int_{-\infty}^{\infty}\left|u_{x}\right|^{2} d x-2 \int_{-\infty}^{\infty} \lambda|u|^{2} d x \\
& \leqslant-2 \lambda^{\prime} \int_{-\infty}^{\infty}|u|^{2} d x
\end{aligned}
$$

in $(0, \infty)$; this yields $(3.3)$.

The estimate (3.3) remains valid for mild solutions, too. Indeed, for any given $u_{0} \in L^{2}(\mathbb{R})$ we may choose a sequence $\left(u_{0, n}\right) \subset H^{3}(\mathbb{R})$, converging to $u_{0}$ in $L^{2}(\mathbb{R})$. Then the corresponding strong solutions $u_{n}$ satisfy the estimates

$$
\left\|u_{n}(t)\right\|_{L^{2}(\mathbb{R})} \leqslant e^{-\lambda_{0} t}\left\|u_{0, n}\right\|_{L^{2}(\mathbb{R})}
$$

for each $n$ and $t \geqslant 0$. Letting $n \rightarrow \infty$ this yields (3.3) because $u_{n}(t) \rightarrow u(t)$ in $L^{2}(\mathbb{R})$ for each fixed $t \geqslant 0$.

The preceding proposition ensures the exponential stability only for dampings which are effective on the whole real line. Our next result allows us to weaken this assumption. Set

$$
c_{p}:=\left(1-\frac{1}{2 p}\right)\left(\frac{2}{p}\right)^{\frac{1}{2 p-1}} \text { for } 1 \leqslant p<\infty .
$$


Proposition 3.3. Let $\lambda \in L^{\infty}(\mathbb{R})$. If there exist a positive number $\lambda_{0}$ and a function $\lambda_{1} \in L^{p}(\mathbb{R})$ for some $1 \leqslant p<\infty$ such that

$$
\lambda \geqslant \lambda_{0}+\lambda_{1} \text { almost everywhere }
$$

and

$$
\left\|\lambda_{1}\right\|_{L^{p}(\mathbb{R})}<\left(\frac{\lambda_{0}}{c_{p}}\right)^{1-\frac{1}{2 p}}
$$

then the problem (3.2) is exponentially stable and its solutions satisfy the decay estimates

$$
\|u(t)\|_{L^{2}(\mathbb{R})} \leqslant e^{-\lambda^{\prime} t}\left\|u_{0}\right\|_{L^{2}(\mathbb{R})} \quad \text { for all } t \geqslant 0
$$

with

$$
\lambda^{\prime}:=\lambda_{0}-c_{p}\left\|\lambda_{1}\right\|_{L^{p}(\mathbb{R})}^{1+\frac{1}{2 p-1}}>0 .
$$

In the sequel we often write $\|\cdot\|_{p}$ instead of $\|\cdot\|_{L^{p}(\mathbb{R})}$.

Proof of Proposition 3.3. For $\lambda_{1}=0$ the proposition reduces to the preceding one. Henceforth we assume that $\left\|\lambda_{1}\right\|_{L^{p}(\mathbb{R})}>0$. In the sequel all integrations take place on $\mathbb{R}$. Hence we omit the integration limits $\pm \infty$ and we write $\|\cdot\|_{p}$ instead of $\|\cdot\|_{L^{p}(\mathbb{R})}$ for brevity.

It suffices to establish the following estimate:

$$
\frac{d}{d t}\|u\|_{2}^{2} \leqslant-2 \lambda^{\prime}\|u\|_{2}^{2}
$$

Taking the real parts and applying a density argument it suffices to consider real-valued smooth solutions. We recall from the preceding proof the following identity:

$$
\frac{d}{d t}\left(\int u^{2} d x\right)=-2\left(\int u_{x}^{2} d x+\int \lambda u^{2} d x\right)
$$

Using elementary estimates, Hölder and interpolation inequalities we have

$$
\begin{aligned}
-\int u_{x}^{2} d x-\int \lambda u^{2} d x & =-\int u_{x}^{2} d x-\int\left(\lambda-\lambda_{1}\right) u^{2} d x-\int \lambda_{1} u^{2} d x \\
& \leqslant-\left\|u_{x}\right\|_{2}^{2}-\lambda_{0}\|u\|_{2}^{2}+\left\|\lambda_{1}\right\|_{p}\|u\|_{2 q}^{2} \\
& \leqslant-\left\|u_{x}\right\|_{2}^{2}-\lambda_{0}\|u\|_{2}^{2}+\left\|\lambda_{1}\right\|_{p}\|u\|_{2}^{\frac{2}{q}}\|u\|_{\infty}^{\frac{2}{p}},
\end{aligned}
$$

where $q$ satisfy $\frac{1}{p}+\frac{1}{q}=1$.

Next we observe that

$$
\|v\|_{\infty}^{2} \leqslant 2\|v\|_{2}\left\|v_{x}\right\|_{2}
$$

for all $v \in H^{1}(\mathbb{R})$. Indeed, if $v \in C_{c}^{\infty}(\mathbb{R})$ and $y \in \mathbb{R}$, then we have the following inequality:

$$
\left|v(y)^{2}\right|=\left|\int_{-\infty}^{y} 2 v v_{x} d x\right| \leqslant 2 \int_{-\infty}^{\infty}|v| \cdot\left|v_{x}\right| d x \leqslant 2\|v\|_{2}\left\|v_{x}\right\|_{2}
$$

proving our estimate for smooth functions. The general case follows by density. 
Using (3.7) and applying the Young inequality we deduce from the preceding inequality for any fixed $\varepsilon>0$ that

$$
\begin{aligned}
-\int u_{x}^{2} d x-\int \lambda u^{2} d x & \leqslant-\left\|u_{x}\right\|_{2}^{2}-\lambda_{0}\|u\|_{2}^{2}+2^{\frac{1}{p}}\left\|\lambda_{1}\right\|_{p}\|u\|_{2}^{\frac{2 p-1}{p}}\left\|u_{x}\right\|_{2}^{\frac{1}{p}} \\
& =-\left\|u_{x}\right\|_{2}^{2}-\lambda_{0}\|u\|_{2}^{2}+\left(\frac{1}{\varepsilon}\left\|\lambda_{1}\right\|_{p}\|u\|_{2}^{\frac{2 p-1}{p}}\right)\left(\varepsilon 2^{\frac{1}{p}}\left\|u_{x}\right\|_{2}^{\frac{1}{p}}\right) \\
& \leqslant-\left\|u_{x}\right\|_{2}^{2}-\lambda_{0}\|u\|_{2}^{2}+\frac{\left(\frac{1}{\varepsilon}\left\|\lambda_{1}\right\|_{p}\|u\|_{2}^{\frac{2 p-1}{p}}\right)^{\frac{2 p}{2 p-1}}}{\frac{2 p}{2 p-1}}+\frac{\left(\varepsilon 2^{\frac{1}{p}}\left\|u_{x}\right\|_{2}^{\frac{1}{p}}\right)^{2 p}}{2 p} .
\end{aligned}
$$

Choosing $\varepsilon$ such that $4 \varepsilon^{2 p}=2 p$, the terms $\left\|u_{x}\right\|_{2}^{2}$ eliminate each other and we obtain that

$$
-\left(\int u_{x}^{2} d x+\int \lambda u^{2} d x\right) \leqslant\|u\|_{2}^{2}\left(-\lambda_{0}+\frac{2 p-1}{2 p}\left(\frac{2}{p}\right)^{\frac{1}{2 p-1}}\left\|\lambda_{1}\right\|_{p}^{\frac{2 p}{2 p-1}}\right)=-\lambda^{\prime}\|u\|_{2}^{2} .
$$

Combining this with (3.6) we obtain (3.5).

\section{Remarks 3.4.}

- We observe that the function $\lambda$ in Proposition 3.3 may have negative values.

- The proposition and its proof remains valid in the limiting case $p=\infty$ under the condition $\left\|\lambda_{1}\right\|_{L^{\infty}(\mathbb{R})}<\lambda_{0}$ with $\lambda^{\prime}:=\lambda_{0}-\left\|\lambda_{1}\right\|_{L^{\infty}(\mathbb{R})}>0$ This is equivalent to Proposition 3.2 above.

\section{Well-posedness for the nonlinear problem}

Since the global well-posedness of the problem

$$
\begin{cases}u_{t}+u_{x x x}-u_{x x}+\lambda u+u u_{x}=0 & \text { in } \mathbb{R} \times(0, \infty), \\ u(0)=u_{0} & \text { in } \mathbb{R},\end{cases}
$$

when $u_{0} \in L^{2}(\mathbb{R})$, may be proved in a standard way, we only give a brief sketch.

First we consider the corresponding linear inhomogeneous initial value problem

$$
\begin{cases}u_{t}+u_{x x x}-u_{x x}+\lambda u=f & \text { in } \mathbb{R} \times(0, T), \\ u(0)=u_{0} & \text { in } \mathbb{R}\end{cases}
$$

for some given $0<T<\infty$. Setting

$$
A:=-\partial_{x}^{3}+\partial_{x}^{2}-\lambda I, \quad D(A)=H^{3}(\mathbb{R}),
$$

it can be written in the form

$$
u_{t}=A u+f, \quad u(0)=u_{0} .
$$

According to Section 2, A generates a strongly continuous semigroup $\{S(t)\}_{t} \geqslant 0$ of contractions in $L^{2}(\mathbb{R})$. Hence for any given $u_{0} \in L^{2}(\mathbb{R}), T>0$ and $f \in L^{1}\left(0, T ; L^{2}(\mathbb{R})\right)$, (4.2) has a unique mild solution $u \in C\left([0, T] ; L^{2}(\mathbb{R})\right)$, given by the formula

$$
u(t)=S(t) u_{0}+\int_{0}^{t} S(t-s) f(s) d s, \quad t \in[0, T],
$$

and depending continuously on the data:

$$
\|u\|_{C\left([0, T] ; L^{2}(\mathbb{R})\right)}:=\sup _{t \in[0, T]}\|u\|_{L^{2}(\mathbb{R})} \leqslant\left\|u_{0}\right\|_{L^{2}(\mathbb{R})}+\|f\|_{L^{1}\left(0, T ; L^{2}(\mathbb{R})\right)} .
$$

In fact, the solution of (4.2) has an additional space regularity. Let us introduce the Banach space

$$
\mathcal{B}=\mathcal{B}_{T}:=C\left([0, T] ; L^{2}(\mathbb{R})\right) \cap L^{2}\left(0, T ; H^{1}(\mathbb{R})\right)
$$


with the norm

$$
\|u\|_{\mathcal{B}}=\|u\|_{C\left([0, T] ; L^{2}(\mathbb{R})\right)}+\left\|\partial_{x} u\right\|_{L^{2}\left(0, T ; L^{2}(\mathbb{R})\right)} .
$$

We have the following

Proposition 4.1. If $u_{0} \in L^{2}(\mathbb{R})$ and $f \in L^{1}\left(0, T ; L^{2}(\mathbb{R})\right)$, then the solution $u$ of (4.2) belongs to $\mathcal{B}$, and

$$
\|u\|_{\mathcal{B}} \leqslant c_{T}\left(\left\|u_{0}\right\|_{L^{2}(\mathbb{R})}+\|f\|_{L^{1}\left(0, T ; L^{2}(\mathbb{R})\right)}\right)
$$

with $c_{T}=2 e^{T\|\lambda\|_{\infty}}$.

Furthermore, the following energy identity holds for all $t \in[0, T]$ :

$$
\begin{aligned}
& \frac{1}{2}\|u(t)\|_{L^{2}(\mathbb{R})}^{2}+\int_{0}^{t}\left\|\partial_{x} u(s)\right\|_{L^{2}(\mathbb{R})}^{2} d s+\int_{0}^{t} \int_{\mathbb{R}} \lambda(x)|u(x, s)|^{2} d x d s \\
& =\frac{1}{2}\left\|u_{0}\right\|_{L^{2}(\mathbb{R})}^{2}+\int_{0}^{t} \int_{\mathbb{R}} f(x, s) u(x, s) d x d s .
\end{aligned}
$$

Now we turn to the nonlinear problem. Let $u_{0} \in L^{2}(\mathbb{R})$. Motivated by the preceding considerations, by a mild solution of (4.1) we mean a function $u \in \mathcal{B}_{T}, T>0$, satisfying

$$
u(t)=S(t) u_{0}-\int_{0}^{t} S(t-s) u(s) \partial_{x} u(s) d s, \quad t \in[0, T] .
$$

By a global mild solution of (4.1) we mean a function $u:[0, \infty) \rightarrow H^{1}(\mathbb{R})$ whose restriction to every bounded interval $[0, T]$ is a mild solution of (4.1).

We have the following

Theorem 4.2. For any given $u_{0} \in L^{2}(\mathbb{R})$ the problem (4.1) has a unique global mild solution.

Furthermore, the following energy identity holds for all $t \geqslant 0$ :

$$
\frac{1}{2}\|u(t)\|_{L^{2}(\mathbb{R})}^{2}+\int_{0}^{t}\left\|\partial_{x} u(s)\right\|_{L^{2}(\mathbb{R})}^{2} d s+\int_{0}^{t} \int_{\mathbb{R}} \lambda(x)|u(x, s)|^{2} d x d s=\frac{1}{2}\left\|u_{0}\right\|_{L^{2}(\mathbb{R})}^{2} .
$$

For the proof we need a lemma (see [20, Proposition 4.1]):

\section{Lemma 4.3.}

(a) If $u \in L^{2}\left(0, T ; H^{1}(\mathbb{R})\right)$, then $M u:=u \partial_{x} u \in L^{1}\left(0, T ; L^{2}(\mathbb{R})\right)$.

(b) If, moreover, $u, v \in \mathcal{B}$, then

$$
\|M u-M v\|_{L^{1}\left(0, T ; L^{2}(\mathbb{R})\right)} \leqslant \sqrt{2} T^{1 / 4}\left(\|u\|_{\mathcal{B}}+\|v\|_{\mathcal{B}}\right)\|u-v\|_{\mathcal{B}} .
$$

Following the ideas contained in [21], using this lemma we may establish the local well posedness using a fixed point argument:

Lemma 4.4. Given $u_{0} \in L^{2}(\mathbb{R})$, the problem (4.1) has a unique mild solution for every sufficiently small $T>0$.

Furthermore, the solution satisfies the identity (4.3) for all $t \in[0, T]$. 
Now the proof of Theorem 4.2 may be completed as follows. The uniqueness follows from the local uniqueness in Lemma 4.4. For the global existence we only have to show that the solution cannot blow up at any finite time $T$.

It follows from Theorem 4.2 that, for each fixed $T>0$, the solution map

$$
\mathcal{A}: L^{2}(\mathbb{R}) \rightarrow \mathcal{B}, \quad u_{0} \mapsto u,
$$

where $u=\mathcal{A} u_{0}$ is the corresponding solution of the problem (4.1), is well defined.

Next we investigate the well-posedness of the nonlinear problem for more regular data. We define for each any fixed $s \in[0,3]$ and $T>0$ the space

$$
\mathcal{B}_{s, T}:=C\left([0, T] ; H^{s}(\mathbb{R})\right) \cap L^{2}\left(0, T ; H^{s+1}(\mathbb{R})\right)
$$

endowed with norm

$$
\|u\|_{\mathcal{B}_{s, T}}:=\sup _{t \in[0, T]}\|u(t)\|_{H^{s}(\mathbb{R})}+\|u\|_{L^{2}\left(0, T ; H^{s+1}(\mathbb{R})\right)} .
$$

In fact, using a method first introduced by Tartar [25] and adapted by Bona and Scott [26], we prove the following result

Theorem 4.5. Let $T>0$ and $\lambda \in H^{1}(\mathbb{R})$. For every $u_{0} \in H^{s}(\mathbb{R}), 0 \leqslant s \leqslant 3$, the nonlinear problem (4.1) admits a unique solution $u$, which belongs to the class $\mathcal{B}_{s, T}$. Also, there exists a continuous function $C: \mathbb{R}^{+} \times(0, \infty) \rightarrow \mathbb{R}^{+}$, nondecreasing in its first variable, such that

$$
\|u\|_{\mathcal{B}_{s, T}} \leqslant C\left(\left\|u_{0}\right\|_{2}, T\right)\left\|u_{0}\right\|_{H^{s}(\mathbb{R})} .
$$

To prove the above theorem we need first to present the cited method and we also need to prove some auxiliary results.

Let $B_{0}$ and $B_{1}$ be two Banach spaces such that $B_{1} \subset B_{0}$ with the inclusion map continuous. Let $f \in B_{0}$ and, for $t \geqslant 0$, define

$$
K(f, t)=\inf _{g \in B_{1}}\left\{\|f-g\|_{B_{0}}+t\|g\|_{B_{1}}\right\} .
$$

For $0<\theta<1$ and $1 \leqslant p \leqslant \infty$, define

$$
\left[B_{0}, B_{1}\right]_{\theta, p}=B_{\theta, p}=\left\{f \in B_{0}:\|f\|_{\theta, p}:=\left(\int_{0}^{\infty} K(f, t)^{p} t^{-\theta p-1} d t\right)^{\frac{1}{p}}<\infty\right\}
$$

with the usual modification for the case $p=\infty$. Then $B_{\theta, p}$ is a Banach space with norm $\|\cdot\|_{\theta, p}$. Given two pairs $\left(\theta_{1}, p_{1}\right)$ and $\left(\theta_{2}, p_{2}\right)$ as above, then $\left(\theta_{1}, p_{1}\right) \prec\left(\theta_{2}, p_{2}\right)$ means

$$
\begin{cases}\theta_{1}<\theta_{2}, & \text { or } \\ \theta_{1}=\theta_{2} & \text { and } p_{1}>p_{2} .\end{cases}
$$

If $\left(\theta_{1}, p_{1}\right) \prec\left(\theta_{2}, p_{2}\right)$ then $B_{\theta_{2}, p_{2}} \subset B_{\theta_{1}, p_{1}}$ with the inclusion map continuous.

The interpolation result is the following.

Theorem 4.6. (See Bona and Scott [26, Theorem 4.3].) Let $B_{0}^{j}$ and $B_{1}^{j}$ be Banach spaces such that $B_{1}^{j} \subset B_{0}^{j}$ with continuous inclusion mappings, $j=1,2$. Let $\alpha$ and $q$ lie in the ranges $0<\alpha<1$ and $1 \leqslant q \leqslant \infty$. Suppose $A$ is a mapping such that

(i) $A: B_{\alpha, q}^{1} \rightarrow B_{0}^{2}$ and for $f, g \in B_{\alpha, q}^{1}$,

$$
\|A f-A g\|_{B_{0}^{2}} \leqslant C_{0}\left(\|f\|_{B_{\alpha, q}^{1}}+\|g\|_{B_{\alpha, q}^{1}}\right)\|f-g\|_{B_{0}^{1}}
$$

and 
(ii) $A: B_{1}^{1} \rightarrow B_{1}^{2}$ and for $h \in B_{1}^{1}$,

$$
\|A h\|_{B_{1}^{2}} \leqslant C_{1}\left(\|h\|_{B_{\alpha, q}^{1}}\right)\|h\|_{B_{1}^{1}},
$$

where $C_{j}: \mathbb{R}^{+} \rightarrow \mathbb{R}^{+}$are continuous nondecreasing functions, $j=0,1$.

Then if $(\theta, p) \geqslant(\alpha, q)$, A maps $B_{\theta, p}^{1}$ into $B_{\theta, q}^{2}$ and for $f \in B_{\theta, p}^{1}$ we have

$$
\|A f\|_{B_{\theta, p}^{2}} \leqslant C\left(\|f\|_{B_{\alpha, q}^{1}}\right)\|f\|_{B_{\theta, p}^{1}},
$$

where $r>0, C(r)=4 C_{0}(4 r)^{1-\theta} C_{1}(3 r)^{\theta}$.

Using classical theory of linear semigroup and a contraction map theorem argument as presented in [26] we establish the following existence theorem for the problem (4.1).

Theorem 4.7. Let $T>0$ and $\lambda \in H^{1}(\mathbb{R})$. For any $u_{0} \in H^{3}(\mathbb{R})$ there exists a unique solution $u$ of (4.1) in the class $\mathcal{B}_{3, T}$ with $u_{t} \in \mathcal{B}_{0, T}$. Also, there exists a continuous function $\beta_{3}: \mathbb{R}^{+} \times(0, \infty) \rightarrow \mathbb{R}^{+}$, nondecreasing in its first variable, such that

$$
\|u\|_{\mathcal{B}_{3, T}} \leqslant \beta_{3}\left(\left\|u_{0}\right\|_{2}, T\right)\left\|u_{0}\right\|_{H^{3}(\mathbb{R})}
$$

The proof of the previous result also requires an adaptation of Lemma 4.3 as follows.

Lemma 4.8. Let $T>0$. For any $u, v \in \mathcal{B}_{3, T}$ such that $u_{t}, v_{t} \in \mathcal{B}_{0, T}$ we have

$$
\frac{1}{2}(u v)_{x} \in W^{1,1}\left(0, T ; L^{2}(\mathbb{R})\right) \text { and } \frac{1}{2}(u v)_{x x} \in L^{2}\left(0, T ; L^{2}(\mathbb{R})\right) .
$$

In addition, the following estimates hold

$$
\left\|\frac{1}{2}(u v)_{x}\right\|_{W^{1,1}\left(0, T ; L^{2}(\mathbb{R})\right)} \leqslant \sqrt{2} T^{\frac{1}{4}}\left(\|u\|_{\mathcal{B}_{3, T}}\|v\|_{\mathcal{B}_{3, T}}+\|u\|_{\mathcal{B}_{3, T}}\left\|v_{t}\right\|_{\mathcal{B}_{0, T}}+\left\|u_{t}\right\|_{\mathcal{B}_{0, T}}\|v\|_{\mathcal{B}_{3, T}}\right)
$$

and

$$
\left\|\frac{1}{2}(u v)_{x x}\right\|_{L^{2}\left(0, T ; L^{2}(\mathbb{R})\right)} \leqslant 2^{\frac{3}{2}} T^{\frac{1}{2}}\|u\|_{\mathcal{B}_{3, T}}\|v\|_{\mathcal{B}_{3, T}} .
$$

Proof of Theorem 4.7. Let $T>0$ be fixed, $\lambda \in H^{1}(\mathbb{R})$ and $u_{0} \in H^{3}(\mathbb{R})$. For $0<\theta \leqslant T$ and $R>0$ define

$$
S_{\theta, R}:=\left\{u \in \mathcal{B}_{3, \theta} \text { such that } u_{t} \in \mathcal{B}_{0, \theta} \text { and }\|u\|_{\mathcal{B}_{3, \theta}}+\left\|u_{t}\right\|_{\mathcal{B}_{0, \theta}} \leqslant R\right\} .
$$

It follows from Lemma 4.8 that $\frac{1}{2}\left(v^{2}\right)_{x} \in W^{1,1}\left(0, \theta ; L^{2}(\mathbb{R})\right)$ and $\frac{1}{2}\left(v^{2}\right)_{x x} \in L^{2}\left(0, \theta ; L^{2}(\mathbb{R})\right)$. Therefore, using classical linear semigroup results, it follows that the linear inhomogeneous problem

$$
\begin{cases}u_{t}+u_{x x x}-u_{x x}+\lambda u=\frac{1}{2}\left(v^{2}\right)_{x} & \text { in } \mathbb{R} \times(0, \theta), \\ u(0)=u_{0} & \text { in } \mathbb{R},\end{cases}
$$

has a unique solution $u$ in the class $\mathcal{B}_{3, \theta}$ such that $u_{t}$ is the solution of

$$
\begin{cases}w_{t}+w_{x x x}-w_{x x}+\lambda w=\frac{1}{2}\left(v^{2}\right)_{x t} & \text { in } \mathbb{R} \times(0, \theta), \\ w(0)=w_{0} & \text { in } \mathbb{R}\end{cases}
$$


with $w_{0}:=-u_{0 x x x}+u_{0 x x}-\lambda u_{0}+\frac{1}{2}\left(v(0)^{2}\right)_{x} \in L^{2}(\mathbb{R})$ and $u_{t} \in \mathcal{B}_{0, \theta}$. Also, there exists a positive constant $c_{3, \theta}$ verifying

$$
\|u\|_{\mathcal{B}_{3, \theta}}+\left\|u_{t}\right\|_{\mathcal{B}_{0, \theta}} \leqslant c_{3, \theta}\left(\left\|u_{0}\right\|_{H^{3}(\mathbb{R})}+\left\|\frac{1}{2}\left(v^{2}\right)_{x}\right\|_{W^{1,1}\left(0, \theta ; L^{2}(\mathbb{R})\right)}+\left\|\frac{1}{2}\left(v^{2}\right)_{x x}\right\|_{L^{2}\left(0, \theta ; L^{2}(\mathbb{R})\right)}\right) .
$$

Therefore, the application $\Gamma$ defined by

$$
v \in S_{\theta, R} \mapsto \Gamma v=\left(u, u_{t}\right) \in \mathcal{B}_{3, \theta} \times \mathcal{B}_{0, \theta},
$$

which associates to each $v \in S_{\theta, R}$ the corresponding solutions $u$ and $u_{t}$, respectively. Also, using Lemma 4.8 and the fact that $c_{3, \theta} \leqslant c_{3, T}$ for every $\theta \leqslant T$, we prove

$$
\|\Gamma v\|_{\mathcal{B}_{3, \theta} \times \mathcal{B}_{0, \theta}} \leqslant c_{3, T}\left\|u_{0}\right\|_{H^{3}(\mathbb{R})}+c_{3, T} 2^{\frac{3}{2}}\left(\theta^{\frac{1}{4}}+\theta^{\frac{1}{2}}\right) R^{2} .
$$

Defining $R=2 c_{3, T}\left\|u_{0}\right\|_{H^{3}(\mathbb{R})}$ and choosing $0<\theta \leqslant T$ such that

$$
c_{3, T} 2^{\frac{5}{2}}\left(\theta^{\frac{1}{4}}+\theta^{\frac{1}{2}}\right) R \leqslant \frac{1}{2}
$$

we conclude that $\|\Gamma v\|_{\mathcal{B}_{3, \theta} \times \mathcal{B}_{0, \theta}} \leqslant R$. For this choice of $R$ and $\theta$, if $u, v \in S_{\theta, R}$ we have

$$
\begin{aligned}
\|\Gamma u-\Gamma v\|_{\mathcal{B}_{3, \theta} \times \mathcal{B}_{0, \theta}} & \leqslant c_{3, \theta}\left(\left\|\frac{1}{2}((u-v)(u+v))_{x}\right\|_{W^{1,1}\left(0, \theta ; L^{2}(\mathbb{R})\right)}+\left\|\frac{1}{2}((u-v)(u+v))_{x x}\right\|_{L^{2}\left(0, \theta ; L^{2}(\mathbb{R})\right)}\right) \\
& \leqslant c_{3, T} 2^{\frac{3}{2}}\left(\theta^{\frac{1}{4}}+\theta^{\frac{1}{2}}\right)\left(\|\vec{u}\|_{\mathcal{B}_{3, \theta} \times \mathcal{B}_{0, \theta}}+\|\vec{v}\|_{\left.\mathcal{B}_{3, \theta} \times \mathcal{B}_{0, \theta}\right)\|\vec{u}-\vec{v}\|_{\mathcal{B}_{3, \theta} \times \mathcal{B}_{0, \theta}}}\right. \\
& \leqslant c_{3, T} 2^{\frac{5}{2}}\left(\theta^{\frac{1}{4}}+\theta^{\frac{1}{2}}\right) R\|\vec{u}-\vec{v}\|_{\mathcal{B}_{3, \theta} \times \mathcal{B}_{0, \theta}} \\
& \leqslant \frac{1}{2}\|\vec{u}-\vec{v}\|_{\mathcal{B}_{3, \theta} \times \mathcal{B}_{0, \theta}},
\end{aligned}
$$

where $\vec{u}=\left(u, u_{t}\right)$, which proves that $\Gamma$ is a contraction. So, $\Gamma$ admits a unique fixed point in $S_{\theta, R}$.

In particular, we have

$$
\sup _{t \in[0, \theta]}\|u(t)\|_{H^{3}(\mathbb{R})}+\sup _{t \in[0, \theta]}\left\|u_{t}(t)\right\|_{L^{2}(\mathbb{R})} \leqslant 2 c_{3, T}\left\|u_{0}\right\|_{H^{3}(\mathbb{R})} .
$$

So, using standards arguments, we may extend $\theta$ to $T$. Finally, the proof is completed defining $\beta_{3}(s)=2 c_{3, T}$ for every $s \in \mathbb{R}^{+}$.

To prove Theorem 4.5 we need a last result.

Proposition 4.9. The solution map (4.4) is locally Lipschitz continuous, i.e., there exists a continuous function $\beta_{0}$ : $\mathbb{R}^{+} \times(0, \infty) \rightarrow \mathbb{R}^{+}$, nondecreasing in its first variable, such that for any $u_{0}, v_{0} \in L^{2}(\mathbb{R})$ we have

$$
\left\|\mathcal{A} u_{0}-\mathcal{A} v_{0}\right\|_{\mathcal{B}} \leqslant \beta_{0}\left(\left\|u_{0}\right\|_{2}+\left\|v_{0}\right\|_{2}, T\right)\left\|u_{0}-v_{0}\right\|_{2} .
$$

Proof. Let $0 \leqslant \theta \leqslant T$ to be determined later. For $u_{0}, v_{0} \in L^{2}(\mathbb{R})$ let $\mathcal{A} u_{0}=u$ and $\mathcal{A} v_{0}=v$ be the corresponding solutions of $(4.1)$ on $[0, T]$. We denote

$$
\mathcal{B}_{\theta}:=C\left([0, \theta] ; L^{2}(\mathbb{R})\right) \cap L^{2}\left(0, \theta ; H^{1}(\mathbb{R})\right) .
$$

The restriction of $u$ and $v$ to $[0, \theta]$ belong to $\mathcal{B}_{\theta}$. Also, $u-v$ is the solution of the linear inhomogeneous problem (4.2) with $f=v v_{x}-u u_{x}$ and initial data $u_{0}-v_{0}$. So, combining Proposition 4.1 with Lemma 4.3 and applying Gronwall's inequality to (4.3) we get

$$
\|u-v\|_{\mathcal{B}_{\theta}} \leqslant c_{\theta}\left\|u_{0}-v_{0}\right\|_{2}+c_{\theta}^{2} \sqrt{2} \theta^{\frac{1}{4}}\left(\left\|u_{0}\right\|_{2}+\left\|v_{0}\right\|_{2}\right)\|u-v\|_{\mathcal{B}_{\theta}},
$$

where $c_{\theta}=2 e^{\theta\|\lambda\|_{\infty}}$. 
Since $0<\theta \leqslant T$, we have $c_{\theta} \leqslant c_{T}$ and choosing $\theta$ verifying

$$
c_{T}^{3} \sqrt{2} \theta^{\frac{1}{4}}\left(\left\|u_{0}\right\|_{2}+\left\|v_{0}\right\|_{2}\right) \leqslant \frac{1}{2},
$$

we obtain

$$
\|u-v\|_{\mathcal{B}_{\theta}} \leqslant 2 c_{T}\left\|u_{0}-v_{0}\right\|_{2} .
$$

Suppose that $2 \theta<T$ and set

$$
\mathcal{B}_{2 \theta}:=C\left([\theta, 2 \theta] ; L^{2}(\mathbb{R})\right) \cap L^{2}\left(\theta, 2 \theta ; H^{1}(\mathbb{R})\right) .
$$

The restrictions of $u$ and $v$ on $[\theta, 2 \theta]$ are the solutions of $(4.1)$ on $[\theta, 2 \theta]$ with respect to the initial data $u(\theta)$ and $v(\theta)$, respectively. Also, proceeding as before we have

$$
\|u-v\|_{\mathcal{B}_{2 \theta}} \leqslant c_{T}\|u(\theta)-v(\theta)\|_{2}+c_{T}^{2} \sqrt{2} \theta^{\frac{1}{4}}\left(\|u(\theta)\|_{2}+\|v(\theta)\|_{2}\right)\|u-v\|_{\mathcal{B}_{2 \theta}} .
$$

Since $\|u(\theta)-v(\theta)\|_{2} \leqslant\|u-v\|_{\mathcal{B}_{\theta}}$, we have

$$
\|u(\theta)-v(\theta)\|_{2} \leqslant 2 c_{T}\left\|u_{0}-v_{0}\right\|_{2},
$$

while applying Gronwall's inequality to (4.3) provides

$$
\|u(\theta)\|_{2}+\|v(\theta)\|_{2} \leqslant c_{\theta}\left(\left\|u_{0}\right\|_{2}+\left\|v_{0}\right\|_{2}\right) .
$$

Gathering these inequalities and observing that $c_{\theta} \leqslant c_{T}$ we conclude

$$
\|u-v\|_{\mathcal{B}_{2 \theta}} \leqslant 2 c_{T}^{2}\left\|u_{0}-v_{0}\right\|_{2}+c_{T}^{3} \sqrt{2} \theta^{\frac{1}{4}}\left(\left\|u_{0}\right\|_{2}+\left\|v_{0}\right\|_{2}\right)\|u-v\|_{\mathcal{B}_{2 \theta}}
$$

and for the choice of $\theta$ we finally have

$$
\|u-v\|_{\mathcal{B}_{2 \theta}} \leqslant 4 c_{T}^{2}\left\|u_{0}-v_{0}\right\|_{2} .
$$

Proceeding similarly, using the number of steps needed to cover the interval $[0, T]$, we conclude the desired result.

Sketch of the proof of Theorem 4.5. Let $T>0$ and $\lambda \in H^{1}(\mathbb{R})$. We define

$$
B_{0}^{1}=L^{2}(\mathbb{R}), \quad B_{0}^{2}=\mathcal{B}_{T}, \quad B_{1}^{1}=H^{3}(\mathbb{R}), \quad B_{1}^{2}=\mathcal{B}_{T, 3}
$$

and let $A$ be the solution map for the nonlinear problem (4.1). Choosing $p=2$ we have, for $0<s<3$ and $\theta=\frac{s}{3}$,

$$
B_{\theta, p}^{2}=\mathcal{B}_{s, T}, \quad B_{\theta, p}^{1}=H^{s}(\mathbb{R}) .
$$

Theorem 4.2 together with Proposition 4.9 provide assumption (i) in the interpolation theorem, while Theorem 4.7 provides assumption (ii). So, if we apply Theorem 4.6, we conclude the proof.

As a consequence, we have the following result which shows that every mild solution of (4.1) is a regular solution when not considering the origin.

Corollary 4.10. Under the assumptions of Theorem 4.5, for any $u_{0} \in L^{2}(\mathbb{R})$, the corresponding solution $u$ of (4.1) belongs to

$$
\mathcal{B}_{3,[\varepsilon, T]}=C\left([\varepsilon, T], H^{3}(\mathbb{R})\right) \cap L^{2}\left(\varepsilon, T ; H^{4}(\mathbb{R})\right)
$$

for every $T>0$ and $0<\varepsilon<T$. 
Proof. Let $T>0$ and $0<\varepsilon<T$. So, for $u_{0} \in L^{2}(\mathbb{R})$, it follows from Theorem 4.5 that the problem (4.1) has a unique solution $u$ in the class $\mathcal{B}_{0, T}$. So, we have $u(t) \in H^{1}(\mathbb{R})$ for almost every $t \in[0, T]$. Let $t_{0} \in(0, \varepsilon)$ such that $u\left(t_{0}\right) \in H^{1}(\mathbb{R})$. Applying Theorem 4.5 with $u_{0}=u\left(t_{0}\right)$, we conclude that the restriction of $u$ to $\left[t_{0}, T\right]$ is the solution

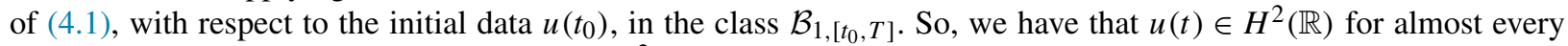
$t \in\left[t_{0}, T\right]$. Let $t_{1} \in\left(t_{0}, \varepsilon\right)$ such that $u\left(t_{1}\right) \in H^{2}(\mathbb{R})$. It follows from Theorem 4.5 that the restriction of $u$ to $\left[t_{1}, T\right]$ is the solution of (4.1), with respect to the initial data $u\left(t_{1}\right)$, in the class $\mathcal{B}_{2,\left[t_{1}, T\right]}$. Finally, since $u(t) \in H^{3}(\mathbb{R})$ for almost every $t \in\left[t_{1}, T\right]$, it follows from Theorem 4.5 that for $t_{2} \in\left(t_{1}, \varepsilon\right)$ such that $u\left(t_{2}\right) \in H^{3}(\mathbb{R})$, the restriction of $u$ to $\left[t_{2}, T\right]$ is the solution of (4.1), with respect to the initial data $u\left(t_{2}\right)$, in the class $\mathcal{B}_{3,\left[t_{2}, T\right]}$ and the result follows.

\section{Exponential decay for the nonlinear problem}

The result established in this section is similar to Proposition 3.3. Hence we only sketch the proof.

We recall the notation

$$
c_{p}:=\left(1-\frac{1}{2 p}\right)\left(\frac{2}{p}\right)^{\frac{1}{2 p-1}} \text { for } 1 \leqslant p<\infty .
$$

Theorem 5.1. Let $\lambda \in L^{\infty}(\mathbb{R})$. If there exist a positive number $\lambda_{0}$ and a function $\lambda_{1} \in L^{p}(\mathbb{R})$ for some $1 \leqslant p<\infty$ such that

$$
\lambda \geqslant \lambda_{0}+\lambda_{1} \quad \text { almost everywhere }
$$

and

$$
\left\|\lambda_{1}\right\|_{L^{p}(\mathbb{R})}<\left(\frac{\lambda_{0}}{c_{p}}\right)^{1-\frac{1}{2 p}},
$$

then the problem (4.1) is exponentially stable and its solutions satisfy the decay estimates

$$
\|u(t)\|_{L^{2}(\mathbb{R})} \leqslant e^{-\lambda^{\prime} t}\left\|u_{0}\right\|_{L^{2}(\mathbb{R})} \text { for all } t \geqslant 0
$$

with

$$
\lambda^{\prime}:=\lambda_{0}-c_{p}\left\|\lambda_{1}\right\|_{L^{p}(\mathbb{R})}^{1+\frac{1}{2 p-1}}>0 .
$$

Remark 5.2. The result and its proof are also valid for $p=\infty$ with $\lambda^{\prime}:=\lambda_{0}-\left\|\lambda_{1}\right\|_{L^{\infty}(\mathbb{R})}$, under the assumption that this value $\lambda^{\prime}$ is positive.

Proof. We omit the variables as well as the integration limits of the spatial variable. We are going to prove the estimate for smooth solutions, the general case follows by density. It suffices to establish the inequality

$$
\frac{d}{d t} \int|u|^{2} d x \leqslant-2 \lambda^{\prime}\|u\|_{2}^{2} .
$$

From the energy identity we have

$$
\begin{aligned}
\frac{d}{d t} \int|u|^{2} d x & =-2 \int\left|\partial_{x} u\right|^{2} d x-2 \int \lambda|u|^{2} d x \\
& \leqslant-2 \int\left|\partial_{x} u\right|^{2} d x-2 \lambda_{0} \int|u|^{2} d x+2 \int \lambda_{1}|u|^{2} d x .
\end{aligned}
$$

Using Hölder's inequality and interpolation we obtain the following estimate:

$$
\begin{aligned}
\int \lambda_{1}|u|^{2} d x & \leqslant 2^{\frac{1}{p}}\left\|\lambda_{1}\right\|_{p}\|u\|_{2}^{\frac{2 p-1}{2}}\left\|\partial_{x} u\right\|_{2}^{\frac{2}{2 p}} \\
& \leqslant c_{p}\left\|\lambda_{1}\right\|_{p}^{\frac{2 p}{2 p-1}}\|u\|_{2}^{2}+\left\|\partial_{x} u\right\|_{2}^{2} .
\end{aligned}
$$

Inequalities (5.3) and (5.2) imply (5.1) as desired. 


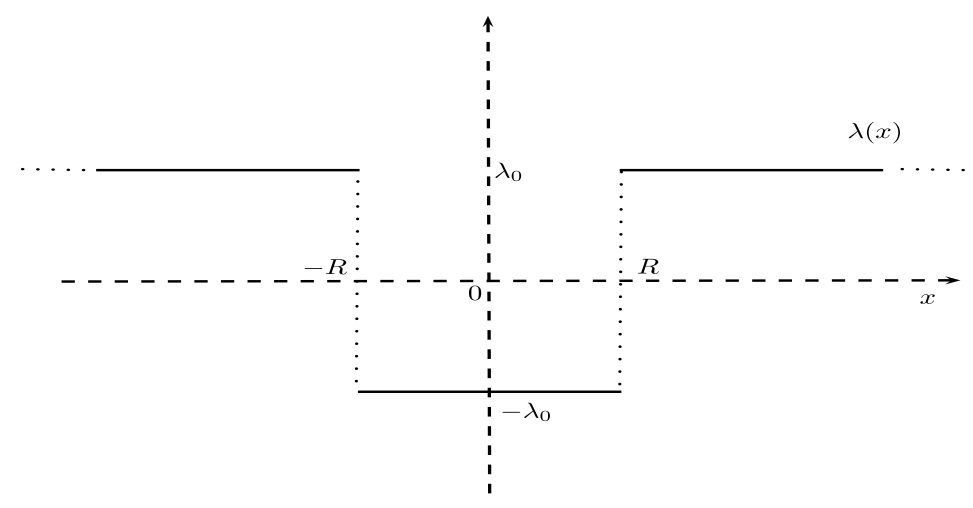

Fig. 1. Graph of $\lambda$.

Corollary 5.3. Let $u_{0} \in L^{2}(\mathbb{R})$ and $\lambda \in L^{\infty}(\mathbb{R})$ satisfying the assumptions of Theorem 5.1. There exists a non-negative continuous function $\alpha_{0}: \mathbb{R}^{+} \times(0, \infty) \rightarrow \mathbb{R}^{+}$such that the corresponding solution $u$ of (4.1), given by Theorem 4.2 , satisfies the inequality

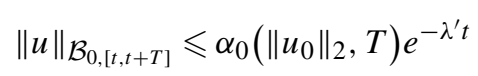

for all $t \geqslant 0$, where we use the notation

$$
\|u\|_{\mathcal{B}_{0,[t, t+T]}}:=\sup _{r \in[t, t+T]}\|u(r)\|_{2}+\left(\int_{t}^{t+T}\left\|u_{x}(r)\right\|_{2}^{2} d r\right)^{\frac{1}{2}} .
$$

Proof. We observe that for every $t \geqslant 0$, the restriction of $u$ to the interval $[t, t+T]$, after a change of variable, is a solution of (4.1) on $[0, T]$, with respect to the initial data $u(t)$. So, applying Theorem 4.5 with $s=0$ and then Theorem 5.1 we conclude that

$$
\begin{aligned}
\|u\|_{\mathcal{B}_{0,[t, t+T]}} & \leqslant C\left(\|u(t)\|_{2}\right)\|u(t)\|_{2} \leqslant C\left(\left\|u_{0}\right\|_{2} e^{-\lambda^{\prime} t}\right)\left\|u_{0}\right\|_{2} e^{-\lambda^{\prime} t} \\
& \leqslant C\left(\left\|u_{0}\right\|_{2}\right)\left\|u_{0}\right\|_{2} e^{-\lambda^{\prime} t} .
\end{aligned}
$$

Example 5.4. Let us consider $\lambda_{0}, R>0$ and $\lambda \in L^{\infty}(\mathbb{R})$ given by

$$
\lambda(x)= \begin{cases}\lambda_{0}, & |x| \geqslant R \\ -\lambda_{0}, & |x|<R\end{cases}
$$

according to Fig. 1.

Let us try to apply Theorem 5.1 with the function $\lambda_{1}: \mathbb{R} \rightarrow \mathbb{R}$ given by

$$
\lambda_{1}(x)= \begin{cases}0, & |x| \geqslant R, \\ -\alpha, & |x|<R,\end{cases}
$$

where $\alpha>0$.

We have $\lambda_{1} \in L^{p}(\mathbb{R})$ with $\left\|\lambda_{1}\right\|_{p}=\alpha(2 R)^{\frac{1}{p}}$ for every $p \in[1, \infty)$.

In order to apply the theorem we must choose $\alpha$ appropriately. We need

$$
\lambda(x) \geqslant \lambda_{0}+\lambda_{1}(x) \quad \text { a.e. in } \mathbb{R} \quad \Longleftrightarrow \quad 2 \lambda_{0} \leqslant \alpha
$$

and

$$
\left\|\lambda_{1}\right\|_{p}<\left(\frac{\lambda_{0}}{c_{p}}\right)^{1-\frac{1}{2 p}} \Longleftrightarrow \alpha^{p}<\frac{1}{2 R}\left(\frac{\lambda_{0}}{c_{p}}\right)^{p-\frac{1}{2}} .
$$


Hence Theorem 5.1 applies with $\lambda_{0}=\alpha / 2$ if

$$
2^{p+1}\left(c_{p}\right)^{p-\frac{1}{2}} \lambda_{0}^{\frac{1}{2}} R<1 \text {. }
$$

This example shows that we may consider indefinite damping mechanisms assuming negative values in arbitrarily big intervals.

\section{Exponential decay for the nonlinear problem in $H^{s}(\mathbb{R})$}

In Section 5 we present an exponential decay result for the solutions of (4.1) in the $L^{2}(\mathbb{R})$ space. Our objective here is to extend this result to any solution of $(4.1)$ in $H^{s}(\mathbb{R})$, with $0 \leqslant s \leqslant 3$.

The main result of this section is the following.

Theorem 6.1. Let $0 \leqslant s \leqslant 3$ and $\lambda \in H^{1}(\mathbb{R})$ satisfying the assumptions of Theorem 5.1. Then there exist a time $T_{0}>0$ and a positive constant $v>0$ such that for every $u_{0} \in H^{s}(\mathbb{R})$ the corresponding solution $u$ of (4.1) satisfies

$$
\|u(t)\|_{H^{s}(\mathbb{R})} \leqslant \beta\left(T_{0},\left\|u_{0}\right\|_{2}\right) e^{-v t}\left\|u_{0}\right\|_{H^{s}(\mathbb{R})}, \quad \text { for } t \geqslant T_{0},
$$

where $\beta:(0, \infty) \times \mathbb{R}^{+} \rightarrow \mathbb{R}^{+}$is a continuous function, nondecreasing in its second variable.

Before proving our main result, let us consider first the linear problem given by

$$
\begin{cases}v_{t}+u_{x x x}-v_{x x}+\lambda v+(v u)_{x}=0 & \text { in } \mathbb{R} \times(0, \infty), \\ v(0)=v_{0} & \text { in } \mathbb{R} .\end{cases}
$$

For this problem we establish the following proposition:

Proposition 6.2. Let $T>0$ and $\lambda \in L^{\infty}(\mathbb{R})$. For each $u \in \mathcal{B}_{0, T}$ and $v_{0} \in L^{2}(\mathbb{R})$, the problem (6.1) admits a unique solution $v$ in the class $\mathcal{B}_{0, T}$. Also, there exist a time $T_{0}>0$ and a continuous function $\sigma:(0, \infty) \times \mathbb{R}^{+} \rightarrow \mathbb{R}^{+}$, nondecreasing in its second variable, such that

$$
\|v\|_{\mathcal{B}_{0, T}} \leqslant \sigma\left(T,\|u\|_{\mathcal{B}_{0, T}}\right)\left\|v_{0}\right\|_{2}
$$

Proof. The proof of the existence follows the steps of Theorem 4.2. Let us prove inequality (6.2). We start by multiplying Eq. (6.1) by $v$ and perform integration by parts. Then, we have (we are going to omit the variables for simplicity)

$$
\begin{aligned}
\frac{d}{d t}\|v\|_{2}^{2}+2\left\|v_{x}\right\|_{2}^{2} & =-2 \int_{\mathbb{R}} \lambda v^{2} d x-\int_{\mathbb{R}}(v u)_{x} v d x \leqslant\|\lambda\|_{\infty}\|v\|_{2}^{2}+\int_{\mathbb{R}} u \frac{1}{2}\left(v^{2}\right)_{x} d x \\
& \leqslant\|\lambda\|_{\infty}\|v\|_{2}^{2}+\|u\|_{2}\left\|\frac{1}{2}\left(v^{2}\right)_{x}\right\|_{2} .
\end{aligned}
$$

Now, integrating over $[0, t]$ for $t \leqslant T$ we will find

$$
\|v(t)\|_{2}^{2}+2 \int_{0}^{t}\left\|v_{x}(s)\right\|_{2}^{2} d s \leqslant 2\|\lambda\|_{\infty} \int_{0}^{t}\|v(s)\|_{2}^{2} d s+\left\|v_{0}\right\|_{2}^{2}+2\|u\|_{\mathcal{B}_{0, T}} \int_{0}^{t}\left\|\frac{1}{2}\left(v(s)^{2}\right)_{x}\right\| d x .
$$

Since

$$
\begin{aligned}
\int_{0}^{t}\left\|\frac{1}{2}\left(v(s)^{2}\right)_{x}\right\|_{2} d t & =\int_{0}^{t}\left\|v(s) v_{x}(s)\right\|_{2} d s \\
& \leqslant \int_{0}^{t}\|v(s)\|_{\infty}\left\|v_{x}(s)\right\|_{2} d s
\end{aligned}
$$




$$
\begin{aligned}
& \leqslant \sqrt{2} \int_{0}^{t}\|v(t)\|_{2}^{\frac{1}{2}}\left\|v_{x}(s)\right\|_{2}^{\frac{3}{2}} d s \\
& \leqslant \sqrt{2}\left(\int_{0}^{t}\|v(s)\|_{2}^{2} d s\right)^{\frac{1}{4}}\left(\int_{0}^{t}\left\|v_{x}(s)\right\|_{2}^{2} d s\right)^{\frac{3}{4}} \\
& \leqslant \frac{\sqrt{2}}{4 \eta^{4}} \int_{0}^{t}\|v(s)\|_{2}^{2} d s+\frac{3 \sqrt{2} \eta^{\frac{4}{3}}}{4} \int_{0}^{t}\left\|v_{x}(s)\right\|_{2}^{2} d s
\end{aligned}
$$

where $\eta=\left(\frac{\sqrt{2}}{3\|u\|_{\mathcal{B}_{0, T}}}\right)^{\frac{3}{4}}$, we arrive at

$$
\|v(t)\|_{2}^{2}+\int_{0}^{t}\left\|v_{x}(s)\right\|_{2}^{2} d s \leqslant\left[2\|\lambda\|_{\infty}+\frac{3^{3}\|u\|_{\mathcal{B}_{0, T}}^{4}}{4}\right] \int_{0}^{t}\left\{\|v(s)\|_{2}^{2}+\int_{0}^{s}\left\|v_{x}(r)\right\|_{2}^{2} d r\right\} d s+\left\|v_{0}\right\|_{2}^{2} .
$$

Finally, employing Gronwall's inequality we conclude

$$
\|v(t)\|_{2}^{2}+\int_{0}^{t}\left\|v_{x}(s)\right\|_{2}^{2} d s \leqslant\left\|v_{0}\right\|_{2}^{2} e^{\left(2\|\lambda\|_{\infty}+\frac{3^{3}\|u\|_{\mathcal{B}_{0, T}}^{4}}{4}\right) t},
$$

for every $t \in[0, T]$. Estimate (6.2) follows directly from the above inequality.

We also need a lemma.

Lemma 6.3. There exists a positive constant c such that the following estimate holds in $H^{3}(\mathbb{R})$ :

$$
\frac{1}{c}\|f\|_{H^{3}(\mathbb{R})} \leqslant\|f\|_{2}+\left\|\partial_{x}^{3} f\right\|_{2} .
$$

Proof. It is sufficient to show that

$$
\left\|\partial_{x}^{i} f\right\|_{2} \leqslant c_{i}\left(\|f\|_{2}+\left\|\partial_{x}^{3} f\right\|_{2}\right)
$$

for $i=1,2$ with suitable constants $c_{i}$. Since we have

$$
\left\|\partial_{x}^{1} f\right\|_{2} \leqslant c_{1}\left\|\partial_{x}^{3} f\right\|_{2}^{\frac{1}{3}}\|f\|_{2}^{\frac{2}{3}} \text { and }\left\|\partial_{x}^{2} f\right\|_{2} \leqslant c_{2}\left\|\partial_{x}^{3} f\right\|_{2}^{\frac{2}{3}}\|f\|_{2}^{\frac{1}{3}}
$$

by the Gagliardo-Nirenberg inequality (see Ponce and Linares [28], p. 50), the required estimates follow by applying Young's inequality:

$$
\left\|\partial_{x}^{1} f\right\|_{2} \leqslant \frac{c_{1}}{3}\left\|\partial_{x}^{3} f\right\|_{2}+\frac{2 c_{1}}{3}\|f\|_{2} \quad \text { and } \quad\left\|\partial_{x}^{2} f\right\|_{2} \leqslant \frac{2 c_{1}}{3}\left\|\partial_{x}^{3} f\right\|_{2}+\frac{c_{2}}{3}\|f\|_{2} .
$$

We may now proceed the proof of the main result.

Proof of Theorem 6.1. Case $s=0$ was proved in Theorem 5.1. Let us assume for a while that the result holds for $s=3$, i.e. there exist a time $T_{0}>0$, a constant $\lambda^{\prime \prime}>0$ and a continuous function $\alpha_{3}:(0, \infty) \times \mathbb{R}^{+} \rightarrow \mathbb{R}^{+}$, nondecreasing in its second variable, such that for every $u_{0} \in H^{3}(\mathbb{R})$ the corresponding solution $u$ satisfy the estimate

$$
\|u(t)\|_{H^{3}(\mathbb{R})} \leqslant \alpha_{3}\left(T_{0},\left\|u_{0}\right\|_{2}\right) e^{-\lambda^{\prime \prime} t}\left\|u_{0}\right\|_{H^{3}(\mathbb{R})}
$$

for every $t \geqslant T_{0}$.

Therefore, for $0<s<3$ and $u_{0} \in H^{s}(\mathbb{R})$, it follows from Theorem 4.5 that there exists a unique solution $u$ of (4.1)

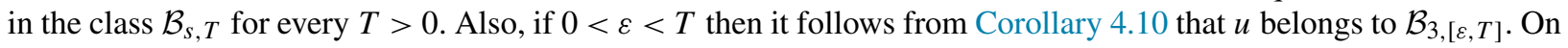
the other hand, using the interpolation inequality (see [27], Eq. (2.43), p. 19) we have 


$$
\|u(t)\|_{H^{s}(\mathbb{R})} \leqslant C\|u(t)\|_{2}^{1-\frac{s}{3}}\|u(t)\|_{H^{3}(\mathbb{R})}^{\frac{s}{3}}, \quad t \geqslant \varepsilon,
$$

where $C>0$ is a constant which comes from interpolation. Combining this estimate with (6.3) and Theorem 5.1 we conclude that

$$
\|u(t)\|_{H^{s}(\mathbb{R})} \leqslant \beta\left(T,\left\|u_{0}\right\|_{2}\right) e^{-v t}\left\|u_{0}\right\|_{H^{s}(\mathbb{R})}, \quad t \geqslant T_{0},
$$

where

$$
\beta\left(T_{0},\left\|u_{0}\right\|_{2}\right)=C \alpha_{3}\left(T_{0},\|u(\varepsilon)\|_{2}\right)^{\frac{s}{3}}\left\|u_{0}\right\|_{2}^{-\frac{s}{3}}\|u(\varepsilon)\|_{H^{3}(\mathbb{R})}^{\frac{s}{3}}
$$

and $v=\lambda^{\prime}\left(1-\frac{s}{3}\right)+\frac{\lambda^{\prime \prime} s}{3}$.

So, it only remains to prove (6.3). We start by considering $u_{0} \in H^{3}(\mathbb{R})$ and $u$ the corresponding solution of (4.1). Therefore, it follows from Lemma 6.3 and Theorem 5.1 that

$$
\begin{aligned}
\frac{1}{c}\|u(t)\|_{H^{3}(\mathbb{R})} & \leqslant\|u(t)\|_{2}+\left\|\partial_{x}^{3} u(t)\right\|_{2} \\
& \leqslant e^{-\lambda^{\prime} t}\left\|u_{0}\right\|_{2}+\left\|\partial_{x}^{3} u(t)\right\|_{2}, \quad \text { for all } t \geqslant 0 .
\end{aligned}
$$

Inequality (6.4) above shows that we just need to establish an exponential estimate for the third derivative in space of $u(t)$. In order to do that, we consider Eq. (4.1) and observe that

$$
\left\|\partial_{x}^{3} u(t)\right\|_{H^{3}(\mathbb{R})} \leqslant\left\|u_{t}(t)\right\|_{2}+\left\|\partial_{x}^{2} u(t)\right\|_{2}+\|\lambda u(t)\|_{2}+\left\|\frac{1}{2}\left(u(t)^{2}\right)_{x}\right\|_{2}, \quad t \geqslant 0 .
$$

First, it is easy to see that

$$
\|\lambda u(t)\|_{2} \leqslant\|\lambda\|_{\infty}\|u(t)\|_{2}, \quad \text { for all } t \geqslant 0
$$

since $\lambda \in H^{1}(\mathbb{R})$.

The nonlinear term is estimated using (3.7) and Young's inequality as described below

$$
\begin{aligned}
\left\|\frac{1}{2}\left(u(t)^{2}\right)_{x}\right\|_{2} & =\left\|u(t) u_{x}(t)\right\|_{2} \\
& \leqslant\|u(t)\|_{\infty}\left\|u_{x}(t)\right\|_{2} \\
& \leqslant 2^{\frac{1}{2}}\|u(t)\|_{2}^{\frac{1}{2}}\left\|\partial_{x}^{1} u(t)\right\|_{2}^{\frac{1}{2}}\left\|\partial_{x}^{1} u(t)\right\|_{2} \\
& \leqslant 2^{-\frac{1}{2}}\|u(t)\|_{2}\left\|\partial_{x}^{1} u(t)\right\|_{2}+2^{-\frac{1}{2}}\left\|\partial_{x}^{1} u(t)\right\|_{2}^{2} \\
& \leqslant 2^{-\frac{3}{2}}\|u(t)\|_{2}^{2}+\left(2^{-\frac{3}{2}}+2^{-\frac{1}{2}}\right)\left\|\partial_{x}^{1} u(t)\right\|_{2}^{2} .
\end{aligned}
$$

Therefore

$$
\begin{aligned}
\left\|\partial_{x}^{3} u(t)\right\|_{2} \leqslant & \left\|u_{t}(t)\right\|_{2}+\left\|\partial_{x}^{2} u(t)\right\|_{2}+\|\lambda\|_{\infty}\|u(t)\|_{2}+2^{-\frac{3}{2}}\|u(t)\|_{2}^{2} \\
& +\left(2^{-\frac{3}{2}}+2^{-\frac{1}{2}}\right)\left\|\partial_{x}^{1} u(t)\right\|_{2}^{2}, \quad t \geqslant 0 .
\end{aligned}
$$

The first and second space-derivatives of $u(t)$ are estimated as in the proof of Lemma 6.3 by using an appropriate Young's inequality

$$
\begin{aligned}
& \left\|\partial_{x}^{1} u(t)\right\|_{2}^{2} \leqslant \frac{2 c_{1}^{2} \gamma^{\frac{3}{2}}}{3}\left\|\partial_{x}^{3} u(t)\right\|_{2}+\frac{c_{1}^{2}}{3 \gamma^{3}}\|u(t)\|_{2}^{4}, \\
& \left\|\partial_{x}^{2} u(t)\right\|_{2} \leqslant \frac{2 c_{2} \gamma^{\frac{3}{2}}}{3}\left\|\partial_{x}^{3} u(t)\right\|_{2}+\frac{c_{2}}{3 \gamma^{3}}\|u(t)\|_{2},
\end{aligned}
$$

where $\gamma$ is a positive real number that will be determinated later. 
Using this estimates we arrive at

$$
\begin{aligned}
\left\|\partial_{x}^{3} u(t)\right\|_{2} \leqslant & \left\|u_{t}(t)\right\|_{2}+\left(\|\lambda\|_{\infty}+\frac{c_{2}}{3 \gamma^{3}}\right)\|u(t)\|_{2}+\frac{c_{1}^{2}}{3 \gamma^{3}}\|u(t)\|_{2}^{4} \\
& +\left(\frac{\left(2^{-\frac{1}{2}}+2^{\frac{1}{2}}\right) c_{1}^{2}}{3}+\frac{2 c_{2}}{3}\right) \gamma^{\frac{3}{2}}\left\|\partial_{x}^{3} u(t)\right\|_{2},
\end{aligned}
$$

from which follows that

$$
c(\gamma)\left\|\partial_{x}^{3} u(t)\right\|_{2} \leqslant\left\|u_{t}(t)\right\|_{2}+\left(\|\lambda\|_{\infty}+\frac{c_{2}}{3 \gamma^{3}}\right)\|u(t)\|_{2}+\frac{c_{1}^{2}}{3 \gamma^{3}}\|u(t)\|_{2}^{4}
$$

where

$$
c(\gamma)=1-\gamma^{\frac{3}{2}} \frac{\left(2^{-\frac{1}{2}}+2^{\frac{1}{2}}\right) c_{1}^{2}+2 c_{2}}{3} .
$$

So, choosing $\gamma=\left(\frac{3}{\left(2^{-\frac{1}{2}}+2^{\frac{1}{2}}\right) c_{1}^{2}+2 c_{2}}\right)^{\frac{2}{3}}$ we have $c(\gamma)=\frac{1}{2}$ and then

$$
\left\|\partial_{x}^{3} u(t)\right\|_{2} \leqslant 2\left\|u_{t}(t)\right\|_{2}+\left(2\|\lambda\|_{\infty}+\frac{2 c_{2}}{3 \gamma^{3}}\right)\|u(t)\|_{2}+\frac{2 c_{1}^{2}}{3 \gamma^{3}}\|u(t)\|_{2}^{4}, \quad t \geqslant 0 .
$$

At this point, we turn our attention to the first time-derivative of $u$. Since Theorem 5.1 can be applied to $u$, the previous inequality shows that we just need to show an exponential estimate for the first time derivative. In order to do that, inspired by the ideas of Proposition 3.9 in Rosier and Zhang's [22], we proceed as follows.

Let $T>0$ be fixed. Since $u$ is given by Theorem 4.5, it follows from Proposition 6.2 that $v=u_{t}$ is the solution of the problem (6.1), with $v_{0}=-u_{0 x x x}+u_{0 x x}-\lambda u_{0}-u_{0} u_{0 x} \in L^{2}(\mathbb{R})$. Furthermore, for every $t \geqslant 0$, it follows from the semigroup property that $v^{t}(s)=v(t+s)$ is the solution of (6.1) with $v^{t}(0)=v(t)$. So, observing that

$$
\begin{aligned}
\left\|v^{t}\right\|_{\mathcal{B}_{0, T}} & =\sup _{s \in[0, T]}\left\|v^{t}(s)\right\|_{2}+\left\|v_{x}^{t}\right\|_{L^{2}(0, T ; L 2(\mathbb{R}))} \\
& =\sup _{s \in[0, T]}\|v(t+s)\|_{2}+\left(\int_{0}^{T}\left\|v_{x}(t+s)\right\|_{2}^{2} d s\right)^{\frac{1}{2}} \\
& =\sup _{r \in[t, t+T]}\|v(r)\|_{2}+\left(\int_{t}^{t+T}\left\|v_{x}(r)\right\|_{2}^{2} d r\right)^{\frac{1}{2}} \\
& :=\|v\|_{\mathcal{B}_{0,[t, t+T]}, \quad t \geqslant 0,}
\end{aligned}
$$

we apply inequality (6.2) and conclude

$$
\|v\|_{\mathcal{B}_{0,[t, t+T]}} \leqslant \sigma\left(T,\|u\|_{\mathcal{B}_{0,[t, t+T]}}\right)\|v(t)\|_{2}, \quad t \geqslant 0 .
$$

Let us now denote $v_{1}(t)=S(t) v_{0}, t \geqslant 0$, where $\{S(t)\}_{t \geqslant 0}$ is the semigroup generated by the linear operator $A$ from Section 4 . Then, $v_{1}$ is the solution of

$$
\begin{cases}v_{1 t}+v_{1 x x x}-v_{1 x x}+\lambda v_{1}=0 & \text { in } \mathbb{R} \times(0, \infty), \\ v_{1}(0)=v_{0} & \text { in } \mathbb{R} .\end{cases}
$$

Since $\lambda$ satisfies the assumptions of Proposition 3.3, we have the following exponential estimate for $v_{1}$

$$
\left\|v_{1}(t)\right\|_{2} \leqslant e^{-\lambda^{\prime} t}\left\|v_{0}\right\|_{2}, \quad t \geqslant 0 .
$$

Now, let us define $v_{2}(t)=\int_{0}^{t} S(t-s)[v(s) u(s)]_{x} d s$, for $t \geqslant 0$. Since $\{S(t)\}_{t \geqslant 0}$ is a semigroup of contractions, we estimate $v_{2}(T)$ in $L^{2}(\mathbb{R})$ using (3.7) and Hölder inequality as follows 


$$
\begin{aligned}
\left\|v_{2}(T)\right\|_{2} & \leqslant \int_{0}^{T}\left\|v_{x}(t) u(t)\right\|_{2}+\left\|v(t) u_{x}(t)\right\|_{2} d t \\
& \leqslant 2^{\frac{1}{2}} \int_{0}^{T}\left\|v_{x}(t)\right\|_{2}\|u(t)\|_{2}^{\frac{1}{2}}\left\|u_{x}(t)\right\|_{2}^{\frac{1}{2}} d t+2^{\frac{1}{2}} \int_{0}^{T}\left\|u_{x}(t)\right\|_{2}\|v(t)\|_{2}^{\frac{1}{2}}\left\|v_{x}(t)\right\|_{2}^{\frac{1}{2}} d t \\
& \leqslant 2^{\frac{3}{2}} T^{\frac{1}{4}}\|v\|_{\mathcal{B}_{0, T}}\|u\|_{\mathcal{B}_{0, T}} .
\end{aligned}
$$

In the last step we have used the estimate

$$
\begin{aligned}
& \int_{0}^{T}\left\|v_{x}(t)\right\|_{2}\|u(t)\|_{2}^{\frac{1}{2}}\left\|u_{x}(t)\right\|_{2}^{\frac{1}{2}} d t \\
& \quad \leqslant\left(\int_{0}^{T}\left\|v_{x}(t)\right\|_{2}^{2} d t\right)^{1 / 2}\left(\int_{0}^{T}\|u(t)\|_{2}^{2} d t\right)^{1 / 4}\left(\int_{0}^{T}\left\|u_{x}(t)\right\|_{2}^{2} d t\right)^{1 / 4} \\
& \quad \leqslant\|v\|_{\mathcal{B}_{0, T}} T^{1 / 4}\|u\|_{\mathcal{B}_{0, T}}^{1 / 2}\|u\|_{\mathcal{B}_{0, T}}^{1 / 2}
\end{aligned}
$$

and the analogous estimate for the second integral.

Therefore, this two estimates combined with the expression of $v$

$$
v(t)=S(t) v_{0}-\int_{0}^{t} S(t-s)[u(s) v(s)]_{x} d s, \quad t \geqslant 0,
$$

leads to the following estimate for $v(T)$

$$
\begin{aligned}
\|v(T)\|_{2} & \leqslant\left\|v_{1}(T)\right\|_{2}+\left\|v_{2}(T)\right\|_{2} \\
& \leqslant e^{-\lambda^{\prime} T}\left\|v_{0}\right\|_{2}+2^{\frac{3}{2}} T^{\frac{1}{4}}\|v\|_{\mathcal{B}_{0, T}}\|u\|_{\mathcal{B}_{0, T}} \\
& \leqslant\left[e^{-\lambda^{\prime} T}+2^{\frac{3}{2}} T^{\frac{1}{4}}\|u\|_{\mathcal{B}_{0, T}} \sigma_{T}\left(\|u\|_{\mathcal{B}_{0, T}}\right)\right]\left\|v_{0}\right\|_{2} .
\end{aligned}
$$

For each $n \in \mathbb{N}$, let us define $y_{n}=v(\cdot, n T)$. If $w_{n}$ is the solution of the problem

$$
\begin{cases}w_{n t}+w_{n x x x}-w_{n x x}+\lambda w_{n}+\left[u^{n T} w_{n}\right]_{x}=0 & \text { in } \mathbb{R} \times(0, \infty), \\ w_{n}(0)=y_{n} & \text { in } \mathbb{R},\end{cases}
$$

where $u^{n T}(x, t)=u(x, t+n T)$, for all $t \geqslant 0$ and $x \in \mathbb{R}$, then $w_{n}(t)=v^{n T}(t)=v(t+n T)$, for every $t \in[0, T]$ and any $n \in \mathbb{N}$. In particular, we have $w_{n}(t)=v^{n T}(T)=v((n+1) T)=y_{n+1}$, for each $n \in \mathbb{N}$, and proceeding as we have done for $v_{1}$ and $v_{2}$ we have

$$
\begin{aligned}
& \left\|y_{n+1}\right\|_{2}=\left\|w_{n}(T)\right\|_{2} \\
& \leqslant\left\|S(T) y_{n}\right\|_{2}+\left\|\int_{0}^{T} S(T-s)\left[u^{n T}(s) w_{n}(s)\right]_{x} d s\right\|_{2} \\
& \leqslant e^{-\lambda^{\prime} T}\left\|y_{n}\right\|_{2}+2^{\frac{3}{2}} T^{\frac{1}{4}}\left\|u^{n T}\right\|_{\mathcal{B}_{0, T}}\left\|v^{n T}\right\|_{\mathcal{B}_{0, T}} \\
& =e^{-\lambda^{\prime} T}\left\|y_{n}\right\|_{2}+2^{\frac{3}{2}} T^{\frac{1}{4}}\|u\|_{\mathcal{B}_{0,[n T,(n+1) T]}}\|v\|_{\mathcal{B}_{0,[n T,(n+1) T]}} \\
& \leqslant e^{-\lambda^{\prime} T}\left\|y_{n}\right\|_{2}+2^{\frac{3}{2}} T^{\frac{1}{4}}\|u\|_{\mathcal{B}_{0,[n T,(n+1) T]}} \sigma\left(T,\|u\|_{\mathcal{B}_{0,[n T,(n+1) T]}}\right)\|v(n T)\|_{2} \\
& \leqslant\left[e^{-\lambda^{\prime} T}+2^{\frac{3}{2}} T^{\frac{1}{4}}\|u\|_{\mathcal{B}_{0,[n T,(n+1) T]}} \sigma\left(T,\|u\|_{\mathcal{B}_{0,[n T,(n+1) T]}}\right)\right]\left\|y_{n}\right\|_{2}
\end{aligned}
$$

for $n=0,1,2, \ldots$ and $T>0$. 
Choosing $T>0$ such that

$$
\alpha_{T}=e^{-\lambda T}<1,
$$

we may determine $\beta>0$ for what

$$
0<r:=\alpha_{T}+2^{\frac{3}{2}} T^{\frac{1}{4}} \beta \sigma(T, \beta)<1 .
$$

Indeed, it is possible since $0<\alpha_{T}<1$ and $\beta \sigma(T, \beta) \rightarrow 0$ as long $\beta \rightarrow 0$. Note that $r$ depends only on $T$, once $\beta$ depends only on $T$.

Now, using Corollary 5.3 we have

$$
\|u\|_{\mathcal{B}_{0,[t, t+T]}} \leqslant \alpha_{0}\left(T,\left\|u_{0}\right\|_{2}\right) e^{-\lambda^{\prime} t}, \quad t \geqslant 0
$$

where the function $\alpha_{0}$ is given by $\alpha_{0}(x, y)=2 e^{\|\lambda\|_{\infty} x} y$ for every $x, y \geqslant 0$. Then, for $N>0$ sufficiently large we have

$$
\alpha_{0}\left(T,\left\|u_{0}\right\|_{2}\right) e^{-\lambda^{\prime} N T} \leqslant \beta .
$$

So, for such value of $N$ we have

$$
\|u\|_{\mathcal{B}_{0,[n T,(n+1) T]}} \leqslant \alpha_{0}\left(T,\left\|u_{0}\right\|_{2}\right) e^{-\lambda^{\prime} N T} \leqslant \beta,
$$

for every $n \in \mathbb{N}$ such that $n \geqslant N$.

Therefore, for $T>0$ and $N>0$ obtained as before we conclude

$$
\left\|y_{n+1}\right\|_{2} \leqslant r\left\|y_{n}\right\|,
$$

for $n \geqslant N$, with $0<r<1$.

Now, using (6.6) and the previous inequality we arrive at

$$
\|v\|_{\mathcal{B}_{0,[(N+k) T,(N+k+1) T]}} \leqslant \sigma\left(T, \alpha_{0}\left(T,\left\|u_{0}\right\|_{2}\right) e^{-\lambda^{\prime} N T}\right) r^{k}\|v(N T)\|_{2} .
$$

Let us denote $T_{0}:=N T$. So, if $t \geqslant T_{0}$ then there exists $k \in \mathbb{N}$ such that $t=(N+k) T+\theta$ with $0 \leqslant \theta<T$. From this, we can write

$$
k=\frac{t-T_{0}-\theta}{T},
$$

and then

$$
\begin{aligned}
\|v(t)\|_{2} & \leqslant\|v\|_{\mathcal{B}_{0,[(N+k) T,(N+k+1) T]}} \\
& \leqslant \sigma\left(T, \alpha_{0}\left(T,\left\|u_{0}\right\|_{2}\right) e^{-\lambda^{\prime} T_{0}}\right) r^{k}\left\|v\left(T_{0}\right)\right\|_{2} \\
& =\sigma\left(T, \alpha_{0}\left(T,\left\|u_{0}\right\|_{2}\right) e^{-\lambda^{\prime} T_{0}}\right) r^{\frac{t-T_{0}-\theta}{T}}\left\|v\left(T_{0}\right)\right\|_{2} .
\end{aligned}
$$

Since $\theta<T$ and $r<1$ we have $r^{\frac{-\theta}{T}}<r^{-1}$ and then

$$
\begin{aligned}
\|v(t)\|_{2} & \leqslant \sigma\left(T, \alpha_{0}\left(T,\left\|u_{0}\right\|_{2}\right) e^{-\lambda^{\prime} T_{0}}\right)\left(\frac{1}{r}\right)^{\frac{-t}{T}} r^{-(N+1)}\left\|v\left(T_{0}\right)\right\|_{2} \\
& =\sigma\left(T, \alpha_{0}\left(T,\left\|u_{0}\right\|_{2}\right) e^{-\lambda^{\prime} T_{0}}\right) e^{-v^{*} t}\left\|v\left(T_{0}\right)\right\|_{2}, \quad t \geqslant T_{0},
\end{aligned}
$$

where $v^{*}=T^{-1} \ln \left(\frac{1}{r}\right)$.

Now, having in mind that $u$ satisfies the last estimate in the proof of Theorem 4.2, we apply (6.2) to conclude

$$
\left\|v\left(T_{0}\right)\right\|_{2} \leqslant \sigma\left(T_{0}, 2 e^{T_{0}\|\lambda\|_{\infty}}\left\|u_{0}\right\|_{2}\right)\left\|v_{0}\right\|_{2} .
$$


Noting that

$$
\begin{aligned}
\left\|v_{0}\right\|_{2} & \leqslant\left\|u_{0 x x x}\right\|_{2}+\left\|u_{0 x x}\right\|_{2}+\left\|\lambda u_{0}\right\|_{2}+\left\|u_{0} u_{0 x}\right\|_{2} \\
& \leqslant\left\|u_{0 x x x}\right\|_{2}+\left\|u_{0 x x}\right\|_{2}+\|\lambda\|_{\infty}\left\|u_{0}\right\|_{2}+\left\|u_{0}\right\|_{2}\left\|u_{0 x}\right\|_{\infty} \\
& \leqslant\left(2+\|\lambda\|_{\infty}\right)\left[\left\|u_{0 x x x}\right\|_{2}+\left\|u_{0 x x}\right\|_{2}+\left\|u_{0}\right\|_{2}\right]+2^{-\frac{1}{2}}\left\|u_{0}\right\|_{2}^{3}+2^{-\frac{1}{2}}\left\|u_{0 x}\right\| \\
& \leqslant\left(2+\|\lambda\|_{\infty}+2^{-\frac{1}{2}}\right)\left[\left\|u_{0 x x x}\right\|_{2}+\left\|u_{0 x x}\right\|_{2}+\left\|u_{0 x}\right\|+\left\|u_{0}\right\|_{2}\right]+2^{-\frac{1}{2}}\left\|u_{0}\right\|_{2}^{3} \\
& \leqslant\left[4\left(2+\|\lambda\|_{\infty}+2^{-\frac{1}{2}}\right)+2^{-\frac{1}{2}}\left\|u_{0}\right\|^{2}\right]\left\|u_{0}\right\|_{H^{3}(\mathbb{R})}
\end{aligned}
$$

and having in mind that $v(t)=u_{t}(t)$ we conclude that

$$
\left\|u_{t}(t)\right\|_{2} \leqslant \alpha_{1}\left(T_{0},\left\|u_{0}\right\|_{2}\right) e^{-v^{*} t}\left\|u_{0}\right\|_{H^{3}(\mathbb{R})}, \quad \text { for } t \geqslant T_{0},
$$

where

$$
\begin{aligned}
\alpha_{1}\left(T_{0},\left\|u_{0}\right\|_{2}\right)= & \sigma\left(T_{0} / N, \alpha_{0}\left(T_{0} / N,\left\|u_{0}\right\|_{2}\right) e^{-\lambda^{\prime} T_{0}}\right) \sigma\left(T_{0}, 2 e^{T_{0}\|\lambda\|_{\infty}}\right) \\
& \times\left(4\left(2+\|\lambda\|_{\infty}+2^{-\frac{1}{2}}\right)+2^{-\frac{1}{2}}\left\|u_{0}\right\|^{2}\right) .
\end{aligned}
$$

Finally, since $u$ is also a solution of $(4.1)$ in $L^{2}(\mathbb{R})$, it follows from Theorem 5.1 that

$$
\|u(t)\|_{2} \leqslant\left\|u_{0}\right\|_{2} e^{-\lambda^{\prime} t}, \quad t \geqslant 0 .
$$

Therefore, combining (6.5), (6.7) and (6.8) we conclude that

$$
\begin{aligned}
\left\|\partial_{x}^{3} u(t)\right\|_{2} & \leqslant 2 \alpha_{1}\left(T_{0},\left\|u_{0}\right\|_{2}\right) e^{-v^{*} t}\left\|u_{0}\right\|_{H^{3}(\mathbb{R})}+\left[2\|\lambda\|_{\infty}+\frac{2 c_{2}}{3 \gamma^{3}}\right] e^{-\lambda^{\prime} t}\left\|u_{0}\right\|_{2}+\frac{2 c_{1}^{2}}{3 \gamma^{3}} e^{-4 \lambda^{\prime} t}\left\|u_{0}\right\|_{2}^{4} \\
& \leqslant \alpha_{2}\left(T_{0},\left\|u_{0}\right\|_{2}\right) e^{-\lambda^{\prime \prime} t}\left\|u_{0}\right\|_{H^{3}(\mathbb{R})}, \quad t \geqslant T_{0},
\end{aligned}
$$

where

$$
\alpha_{2}\left(T_{0},\left\|u_{0}\right\|_{2}\right)=2 \alpha_{1}\left(T_{0},\left\|u_{0}\right\|_{2}\right)+\left[2\|\lambda\|_{\infty}+\frac{2 c_{2}}{3 \gamma^{3}}\right]+\frac{2 c_{1}^{2}}{3 \gamma^{3}}\left\|u_{0}\right\|_{2}^{3}
$$

and $\lambda^{\prime \prime}=\min \left\{v^{*}, \lambda^{\prime}\right\}$.

Therefore, replacing the last estimate in (6.4) it yields

$$
\frac{1}{c}\|u(t)\|_{H^{3}(\mathbb{R})} \leqslant e^{-\lambda^{\prime} t}\left\|u_{0}\right\|_{2}+\alpha_{2}\left(T_{0},\left\|u_{0}\right\|_{2}\right) e^{-\lambda^{\prime \prime} t}\left\|u_{0}\right\|_{H^{3}(\mathbb{R})} .
$$

Inequality (6.3) then follows from the previous one just by choosing

$$
\alpha_{3}\left(T_{0},\left\|u_{0}\right\|_{2}\right)=c\left(1+\alpha_{2}\left(T_{0},\left\|u_{0}\right\|_{2}\right)\right) .
$$

\section{Acknowledgements}

The authors thank the anonymous referee for his valuable suggestion to extend the results of the first version of our work to the $H^{s}$ case and Professor Lionel Rosier for fruitful discussions.

\section{References}

[1] C. Amick, J. Bona, M. Schonbek, Decay of solutions of some nonlinear wave equations, J. Differ. Equ. 81 (1989) 1-49.

[2] J.L. Bona, S.M. Sun, B.-Y. Zhang, Forced oscillations of a damped Korteweg-de Vries equation in a quarter plane, Commun. Contemp. Math. 5 (2003) 369-400.

[3] B.A. Bubnov, Solvability in the large of nonlinear boundary-value problems for the Korteweg-de Vries equation in a bounded domain, Differ. Uravn. 16 (1980) 34-41.English transl. in: Differ. Equ. 16 (1980) 24-30.

[4] M.M. Cavalcanti, V.N. Domingos Cavalcanti, A. Faminskii, F.M.A. Natali, Decay of solutions to damped Korteweg-de Vries type equation, Appl. Math. Optim. 65 (2012) 221-251.

[5] T. Dlotko, The generalized Korteweg-de Vries-Burgers equation in $H^{2}(\mathbb{R})$, Nonlinear Anal. 74 (2011) 721-732. 
[6] A.V. Faminskii, N.A. Larkin, Initial-boundary value problems for quasilinear dispersive equations posed on a bounded interval, Electron. J. Differ. Equ. (2010), 1, 20 pp.

[7] A.V. Faminskii, N.A. Larkin, Odd order evolution equations posed on a bounded interval, Bol. Soc. Parana. Mat. 28 (2010) 67-77.

[8] O. Goubet, J. Shen, On the dual Petrov-Galerkin formulation of the KdV equation, Adv. Differ. Equ. 12 (2007) 817-830.

[9] N. Hyayashi, E. Kaikina, J.G. Zavala, On the boundary-value problem for the Korteweg-de Vries equation, R. Soc. Lond. Proc. Ser. A Math. Phys. Eng. Sci. 459 (2003) 2861-2884.

[10] V. Komornik, D.L. Russel, B.-Y. Zhang, Stabilization de l'équation de Korteweg-de Vries, C. R. Acad. Sci. Paris, Sér. I Math. 312 (1991) $841-843$.

[11] V. Komornik, On the stabilization of the Korteweg-de Vries equation, Bol. Soc. Parana. Mat. (3) 28 (2) (2010) $33-48$.

[12] N.A. Larkin, Korteweg-de Vries and Kuramoto-Sivashinsky equations in bounded domains, J. Math. Anal. Appl. 297 (2004) $169-185$.

[13] N.A. Larkin, Modified KdV equation with a source term in a bounded domain, Math. Methods Appl. Sci. 29 (2006) $751-765$.

[14] C. Laurent, L. Rosier, B.-Y. Zhang, Control and stabilization of the Korteweg-de Vries equation on a periodic domain, Commun. Partial Differ. Equ. 35 (2010) 707-744.

[15] F. Linares, A.F. Pazoto, On the exponential decay of the critical generalized Korteweg-de Vries equation with localized damping, Proc. Am. Math. Soc. 135 (2007) 1515-1522.

[16] F. Linares, A.F. Pazoto, Asymptotic behavior of the Korteweg-de Vries equation posed in a quarter plane, J. Differ. Equ. 833 (2009) $1342-1353$.

[17] G.P. Menzala, C.F. Vasconcellos, E. Zuazua, Stabilization of the Korteweg-de Vries equation with localized damping, J. Q. Appl. Math. 60 (2002) 111-129.

[18] A.F. Pazoto, Unique continuation and decay for the Korteweg-de Vries equation with localized damping, ESAIM Control Optim. Calc. Var. 11 (2005) 473-486.

[19] A.F. Pazoto, L. Rosier, Uniform stabilization in weighted Sobolev spaces for the KdV equation posed on the half-line, Discrete Contin. Dyn. Syst., Ser. B 14 (2010) 1511-1535.

[20] L. Rosier, Exact boundary controllability for the Korteweg-de Vries equation on a bounded domain, ESAIM Control Optim. Calc. Var. 2 (1997) 33-55.

[21] L. Rosier, A.F. Pazoto, Uniform stabilization in weighted Sobolev spaces for the KdV equation posed on the half-line, Discrete Contin. Dyn. Syst., Ser. B 14 (2010) 1511-1535.

[22] L. Rosier, B.-Y. Zhang, Global stabilization of the generalized Korteweg-de Vries equation posed on a finite domain, SIAM J. Control Optim. 3 (2006) 927-956.

[23] L. Rosier, B.-Y. Zhang, Control and stabilization of the Korteweg-de Vries equation: recent progresses, J. Syst. Sci. Complex. 22 (2009) 647-682.

[24] D.L. Russell, B.-Y. Zhang, Exact controllability and stabilizability of the Korteweg-de Vries equation, Trans. Am. Math. Soc. 348 (1996) $3643-3672$.

[25] L. Tartar, Interpolation non linéaire et régularité, J. Funct. Anal. 9 (1972) 469-489.

[26] J.L. Bona, L.R. Scott, Solutions of Korteweg-de Vries equation in fractional order Sobolev spaces, Duke Math. J. 43 (1976) 87-99.

[27] J.-L. Lions, E. Magenes, Non-Homogeneous Boundary Value Problems and Applications. Vol. I, translated from French by P. Kenneth, Grundlehren Math. Wiss., vol. 181, Springer-Verlag, New York, Heidelberg, 1972, xvi+357 pp.

[28] F. Linares, G. Ponce, Introduction to Nonlinear Dispersive Equations, Universitext, Springer-Verlag, New York, ISBN 978-0-387-84898-3, 2009, xii+256 pp. 